\title{
Bose enhancement, the Liouville effective action, and the high multiplicity tail in $p-A$ collisions
}

\author{
Alex Kovner \\ Physics Department, University of Connecticut, 2152 Hillside Road, Storrs, Connecticut 06269, USA \\ and Theoretical Physics Department, CERN, CH-1211 Geneve 23, Switzerland \\ Vladimir V. Skokov \\ RIKEN/BNL, Brookhaven National Laboratory, Upton, New York 11973, USA
}

(Received 5 June 2018; published 5 July 2018)

\begin{abstract}
In the framework of the dense-dilute color glass condensate approach, we study fluctuations in the multiplicity of produced particles in $p-A$ collisions. We show that the leading effect that drives the fluctuations is the Bose enhancement of gluons in the proton wave function. We explicitly calculate the moment generating function that resums the effects of Bose enhancement. We show that it can be understood in terms of the Liouville effective action for the composite field, which is identified with the fluctuating density, or saturation momentum of the proton. The resulting probability distribution turns out to be very close to the $\gamma$ distribution. We also calculate the first correction to this distribution, which is due to pairwise Hanbury Brown-Twiss correlations of produced gluons.
\end{abstract}

DOI: $10.1103 /$ PhysRevD.98.014004

\section{INTRODUCTION}

The study of correlations in $p-A$ and $p-p$ collisions has been a very active area in the last years due to the observation of the ridge correlations at the LHC. Since the ridge signal is much more pronounced in high multiplicity events, it is very important to understand the origin of the multiplicity fluctuation and especially the high multiplicity tail of the distribution; see Refs. [1-4] for the most recent experimental studies. ${ }^{1}$

In the present paper, we address this question in the framework of the color glass condensate (CGC) approach. We calculate the multiplicity momentum generating function, using McLerran-Venugopalan (MV) model for the projectile and assuming that the target is very dense. The latter assumption allows us to employ the factorizable form for the averages of Wilson lines, as explained in Ref. [9]. We show that the main contribution to multiplicity fluctuations arises form the Bose enhancement (BE) of gluons in the projectile wave function (see Ref. [10], providing the interpretation of the correlations in the projectile in terms of BE; a related effect of Pauli blocking for quarks is

\footnotetext{
${ }^{1}$ See also Refs. [5-8] for the experimental and theoretical studies of heavy flavor production in high multiplicity collisions of small systems.

Published by the American Physical Society under the terms of the Creative Commons Attribution 4.0 International license. Further distribution of this work must maintain attribution to the author(s) and the published article's title, journal citation, and DOI. Funded by SCOAP ${ }^{3}$.
}

discussed in Ref. [11]). This effect produces fluctuations that are not suppressed by the factor of the area of the projectile. We are able to resum the $\mathrm{BE}$ contributions exactly in the multiplicity generating function. The resulting distribution turns out to be $\gamma$ distribution, which for large moments practically coincides with the negative binomial distribution.

The other important effect in correlated gluon production is the Hanbury Brown-Twiss (HBT) effect. As discussed at length in the recent literature [12], it is the leading cause for the angular correlations of produced gluons in the CGC approach. Its contribution to the total multiplicity, on the other hand, is suppressed relative to that of $\mathrm{BE}$, as the correlated peak that it produces is very narrow. Nevertheless, we identify the contributions to the multiplicity generating function due to the HBT within our calculational framework and calculate corrections induced by it.

We note that a calculation along similar lines was undertaken some years ago in Ref. [13]; see also Refs. [14-16] for numerical calculations and comparison to experimental data and Ref. [17] for a higher-order correction to a MV model. There are, however, significant differences between our approach and that of Ref. [13]. In particular, Ref. [13] treated both the projectile and the target as dilute. It turns out that the large density effects of the target suppress half of the contributions considered as leading in Ref. [13]. Additionally, the HBT contributions were included in the analysis of Ref. [13]. The resulting multiplicity distribution we obtain is somewhat different from that in Ref. [13]. 
The structure of this paper is the following. In Sec. II, we lay out the general framework of the calculation and perform the averaging over the projectile wave function using the MV model. In Sec. III, we make a detour and consider specifically the second moment of the multiplicity distribution, also known as the inclusive two-gluon cross section. We show explicitly that $\mathrm{BE}$ leads to the largest contribution to this moment and that this contribution is not suppressed by a power of area relative to the square of the single inclusive cross section. The argument is very similar to that in Ref. [13] except for the differences alluded to above due to the dense nature of the target. A similar observation was made also in Ref. [18]. In Sec. IV, we calculate in closed form the momentum generating function that resums all $\mathrm{BE}$ contributions. We also relate it to the constrained effective potential approach proposed in Ref. [19] and show that our result for the momentum generating function is identical to the Liouville theory for the "composite field" discussed in Ref. [19]. In Sec. V, we consider the HBT corrections to the distribution and provide a closed expression that resums the leading correction. Finally, Sec. VI contains a short discussion of our results.

\section{GENERATING FUNCTION}

Our calculations will be performed within the densedilute CGC framework. In this approach, the number of produced gluons for a given configuration of the projectile (proton) and a target (nucleus) is given by [20-22]

$$
\begin{aligned}
\left.\frac{d N}{d^{2} k d y}\right|_{\rho_{\mathrm{p}}, \rho_{\mathrm{t}}}= & \frac{2 g^{2}}{(2 \pi)^{3}} \int \frac{d^{2} q}{(2 \pi)^{2}} \frac{d^{2} q^{\prime}}{(2 \pi)^{2}} \Gamma\left(\underline{k}, \underline{q}, \underline{q}^{\prime}\right) \rho_{\mathrm{p}}^{a}\left(-\underline{q^{\prime}}\right) \\
& \times\left[U^{\dagger}\left(\underline{k}-\underline{q^{\prime}}\right) U(\underline{k}-\underline{q})\right]_{a b} \rho_{\mathrm{p}}^{b}(\underline{q}),
\end{aligned}
$$

where the square of Lipatov vertex is

$$
\Gamma\left(\underline{k}, \underline{q}, \underline{q}^{\prime}\right)=\left(\frac{\underline{q}}{q^{2}}-\frac{\underline{k}}{k^{2}}\right) \cdot\left(\frac{\underline{q}^{\prime}}{q^{\prime 2}}-\frac{\underline{k}}{k^{2}}\right) .
$$

Here, $\rho_{\mathrm{p}}$ is a given configuration of the color charged density in the projectile, and $U$ is the eikonal scattering matrix - the adjoint Wilson line-for scattering of a single gluon on the target. The target Wilson lines depend on the target color sources, $\rho_{\mathrm{t}}$; we suppress this in our notation.

The single inclusive and double inclusive production in this approach are given by

$$
\frac{d N}{d^{2} k d y}=\left\langle\left\langle\left.\frac{d N}{d^{2} k d y}\right|_{\rho_{\mathrm{p}}, \rho_{\mathrm{t}}}\right\rangle_{\mathrm{p}}\right\rangle_{\mathrm{t}}
$$

and $\frac{d^{2} N}{d^{2} k_{1} d y_{1} d^{2} k_{2} d y_{2}}=\left\langle\left\langle\left.\left.\frac{d N}{d^{2} k_{1} d y_{1}}\right|_{\rho_{\mathrm{p}}, \rho_{\mathrm{t}}} \frac{d N}{d^{2} k_{2} d y_{2}}\right|_{\rho_{\mathrm{p}}, \rho_{\mathrm{t}}}\right\rangle_{\mathrm{p}}\right\rangle$,

where the averaging is performed over the projectile and target color charge configurations:

$$
\left\langle O\left(\rho_{\mathrm{p}}\right)\right\rangle_{\mathrm{p}}=\frac{1}{Z_{\mathrm{p}}} \int \mathcal{D} \rho_{\mathrm{p}} W_{\mathrm{p}}\left(\rho_{\mathrm{p}}\right) O\left(\rho_{\mathrm{p}}\right)
$$

and

$$
\left\langle O\left(\rho_{\mathrm{t}}\right)\right\rangle_{\mathrm{t}}=\frac{1}{Z_{\mathrm{t}}} \int \mathcal{D} \rho_{\mathrm{t}} W_{\mathrm{t}}\left(\rho_{\mathrm{t}}\right) O\left(\rho_{\mathrm{t}}\right)
$$

The normalization factors, $Z_{\mathrm{p}, \mathrm{t}}$, are fixed so that

$$
\langle 1\rangle_{\mathrm{p}}=\langle 1\rangle_{\mathrm{t}}=1 .
$$

In general, $m$-particle production is

$$
\begin{aligned}
& \frac{d^{m} N}{d^{2} k_{1} d y_{1} d^{2} k_{2} d y_{2} \ldots d^{2} k_{m} d y_{m}} \\
& =\left\langle\left\langle\left.\left.\left.\frac{d N}{d^{2} k_{1} d y_{1}}\right|_{\rho_{\mathrm{p}}, \rho_{\mathrm{t}}} \frac{d N}{d^{2} k_{2} d y_{2}}\right|_{\rho_{\mathrm{p}}, \rho_{\mathrm{t}}} \ldots \frac{d N}{d^{2} k_{m} d y_{m}}\right|_{\rho_{\mathrm{p}}, \rho_{\mathrm{t}}}\right\rangle_{\mathrm{p}}\right\rangle_{\mathrm{t}}
\end{aligned}
$$

Instead of computing the moments of inclusive particle number fluctuations, we evaluate the moment generating function (see, e.g., Ref. [23])

$$
G(t)=\left\langle\left\langle\exp \left[\left.t \int_{k_{\min }} d^{2} k \frac{d N}{d^{2} k d y}\right|_{\rho_{\mathrm{p}}, \rho_{\mathrm{t}}}\right]\right\rangle_{\mathrm{p}}\right\rangle_{\mathrm{t}},
$$

where we introduced an arbitrary $k_{\min } \gg \Lambda_{\mathrm{QCD}}$. The moments of the distribution are obviously obtained from $G(t)$ by differentiating with respect to $t$ at $t=0$.

To calculate the generating function, we have to specify the distribution of the sources in the projectile and in the target. For the projectile, we will use the simple Gaussian MV model specified by

$$
\left\langle\rho_{\mathrm{p}}^{a}(\underline{p}) \rho_{\mathrm{p}}^{b}(\underline{k})\right\rangle_{\mathrm{p}}=(2 \pi)^{2} \mu_{\mathrm{p}}^{2}(p) \delta(\underline{p}+\underline{k}) \delta^{a b},
$$

which corresponds to the weight functional

$W_{\mathrm{p}}\left(\rho_{\mathrm{p}}\right)=\exp \left(-\int \frac{d^{2} q}{(2 \pi)^{2}} \rho_{\mathrm{p}}^{a}(-\underline{q}) \frac{1}{2 \mu_{\mathrm{p}}^{2}(q)} \rho_{\mathrm{p}}^{a}(\underline{q})\right)$.

Note that the structure of the $\rho_{\mathrm{p}}$ correlator means translational invariance of the projectile wave function in the transverse plane. This assumption is only reasonable if we concentrate on momenta of produced particles larger than 
the inverse radius of the projectile. Thus, in the following, we will always assume $k_{\min }>1 / R$.

The averaging over the Wilson lines of the target will be performed in the approximation articulated recently in Ref. [12]. Any product of Wilson lines is factored into pairs with the basic Wick contraction

$\left\langle U_{a b}(\underline{p}) U_{c d}(\underline{q})\right\rangle_{\mathrm{t}}=\frac{(2 \pi)^{2}}{N_{c}^{2}-1} \delta_{a c} \delta_{b d} \delta(\underline{p}+\underline{q}) D(\underline{p})$.

Here, the adjoint dipole amplitude is defined as

$$
D(p)=\frac{1}{N_{c}^{2}-1} \int d^{2} x e^{i x \cdot p}\left\langle\operatorname{tr}\left[U^{\dagger}(x) U(0)\right]\right\rangle_{\mathrm{t}} .
$$

As explained in Ref. [12], this approximation is appropriate for the dense regime. It collects all terms in the $n$-particle cross section that have the leading dependence on the area of the projectile. The approximation only includes terms that contain "small-size" color singlets in the projectile propagating through the target. Any nonsinglet state that in the transverse plane is removed by more than $1 / Q_{s}$ from other propagating partons must have a vanishing $S$ matrix on the dense (black disk) target. On the other hand, if the singlet state contains more than two partons, one loses a power of the area when integrating over the coordinates of the partons. Thus, the leading contribution in the black disk limit is the one in which only dipole contribution to the $S$ matrix should be accounted for. The same approximation for the quadrupole amplitude has been used previously in Ref. [18], in which its consistency with explicit modeling of the Wilson line correlators via the MV model has been verified.

Note that this averaging procedure for the target is formally (disregarding subtleties related to the definition of the Haar measure) equivalent to the following form of the weight functional:

$$
W_{t}[U]=\exp \left\{-\frac{1}{2} \int \frac{d^{2} q}{(2 \pi)^{2}} \frac{1}{D(q)} \operatorname{tr}\left[U^{\dagger}(q) U(q)\right]\right\}
$$

\section{A. Projectile averaging}

We now consider the calculation of the generating function:

$$
\begin{aligned}
G(t)= & \int D \rho_{\mathrm{p}} D U \exp \left[-\int \frac{d^{2} q}{(2 \pi)^{2}}\left(\rho_{\mathrm{p}}^{a}(-\underline{q}) \frac{1}{2 \mu_{\mathrm{p}}^{2}(q)} \rho_{\mathrm{p}}^{a}(\underline{q})\right.\right. \\
& \left.+\frac{1}{2 D(q)} \operatorname{tr}\left[U^{\dagger}(q) U(-q)\right]\right) \\
& \left.+\left.t \int_{k_{\min }} d^{2} k \frac{d N}{d^{2} k d y}\right|_{\rho_{\mathrm{p}}, \rho_{\mathrm{t}}}\right] .
\end{aligned}
$$

We notice immediately that, since $\frac{d N}{d^{2} k d v}$ is quadratic in both $\rho_{\mathrm{p}}$ and $U$ and both the corresponding weight functionals are Gaussian as well, we can integrate over one of these "fields" exactly. We choose to integrate first over $\rho_{\mathrm{p}}$. The result of this integration is

$$
\begin{aligned}
G(t)= & \int D U \exp \left[-\int \frac{d^{2} q}{(2 \pi)^{2}} \frac{1}{2 D(q)} \operatorname{tr}\left[U^{\dagger}(q) U(-q)\right]\right. \\
& \left.-\frac{1}{2} \operatorname{tr} \ln [1-t M]\right]
\end{aligned}
$$

where the operator $M$ is defined by its matrix elements

$$
\begin{aligned}
M_{a b}\left(q^{\prime}, q\right)= & \frac{4 g^{2}}{(2 \pi)^{3}} \mu^{2}(q) \int_{k_{\min }} \frac{d^{2} k}{(2 \pi)^{2}} \Gamma\left(k, q, q^{\prime}\right) \\
& \times\left[U^{\dagger}\left(\underline{k}-\underline{q}^{\prime}\right) U(\underline{k}-\underline{q})\right]_{a b} .
\end{aligned}
$$

At this point, we need to make some approximations in order to perform the remaining functional integral over $U$. In the next section, we will consider more closely the twogluon production cross section, which will help us understand the systematics of the leading contributions to the multiplicity fluctuations and to devise the appropriate approximation that sums these leading contributions.

\section{DOUBLE INCLUSIVE PRODUCTION: DISSECTING DIFFERENT CONTRIBUTIONS}

To get some insight of which effects contribute the most to the multiplicity fluctuations, we make a short detour and consider the double inclusive gluon production,

$$
\frac{d^{2} N}{d^{2} k_{1} d y_{1} d^{2} k_{2} d y_{2}}=\left\langle\left\langle\left.\left.\frac{d N}{d^{2} k_{1} d y_{1}}\right|_{\rho_{\mathrm{p}}, \rho_{\mathrm{t}}} \frac{d N}{d^{2} k_{2} d y_{2}}\right|_{\rho_{\mathrm{p}}, \rho_{\mathrm{t}}}\right\rangle_{\mathrm{p}}\right\rangle \text {. }
$$

\section{A. Dipole contribution}

First, consider the average with respect to projectile inside each factor $\left.\frac{d N}{d^{2} k d y}\right|_{\rho_{\mathrm{p}}, \rho_{\mathrm{t}}}$, that is,

$$
\begin{aligned}
& {\left[\frac{d^{2} N}{d^{2} k_{1} d y_{1} d^{2} k_{2} d y_{2}}\right]_{\text {Dipole }}} \\
& \quad=\left\langle\left\langle\left.\frac{d N}{d^{2} k_{1} d y_{1}}\right|_{\rho_{\mathrm{p}}, \rho_{\mathrm{t}}}\right\rangle_{\mathrm{p}}\left\langle\left.\frac{d N}{d^{2} k_{2} d y_{2}}\right|_{\rho_{\mathrm{p}}, \rho_{\mathrm{t}}}\right\rangle_{\mathrm{p}}\right\rangle .
\end{aligned}
$$

The projectile averaging gives

$$
\begin{aligned}
\left\langle\left.\frac{d N}{d^{2} k_{1} d y_{1}}\right|_{\rho_{p}, \rho_{\mathrm{t}}}\right\rangle_{\mathrm{p}}= & \frac{2 g^{2}}{(2 \pi)^{3}} \int \frac{d^{2} q}{(2 \pi)^{2}} \mu_{\mathrm{p}}^{2}(\underline{q}) \Gamma\left(\underline{k_{1}}, \underline{q}, \underline{q}\right) \\
& \times \operatorname{tr}\left[U^{\dagger}\left(\underline{k}_{1}-\underline{q}\right) U\left(\underline{k}_{1}-\underline{q}\right)\right] .
\end{aligned}
$$


The subsequent target averages give two distinct contributions. The first one involves target contractions inside each single inclusive factor and is disconnected. It reproduces the square of the single-gluon production probability and is of no interest to us. The connected contribution is

$$
\begin{aligned}
& \left\langle\operatorname{tr}\left[U^{\dagger}\left(\underline{k}_{1}-\underline{q}^{\prime}\right) U\left(\underline{k}_{1}-\underline{q}\right)\right] \operatorname{tr}\left[U^{\dagger}\left(\underline{k}_{2}-\underline{q}^{\prime}\right) U\left(\underline{k}_{2}-\underline{p}\right)\right]\right\rangle_{\mathrm{t}}^{\mathrm{conn} .} \\
& \quad=2 S_{\perp} \delta\left(\underline{k}_{1}+\underline{k}_{2}-\underline{q}-\underline{p}\right) D^{2}\left(\underline{k}_{1}-\underline{q}\right) .
\end{aligned}
$$

The result is of order $N_{c}^{0}$. As we will see in the following, the contractions of $\rho$ that break the factors of $\left.\frac{d N}{d^{2} k d y}\right|_{\rho_{\mathrm{p}}, \rho_{\mathrm{t}}}$ are of order $N_{c}^{2}$ and thus are more important. The contribution from Eq. (21) can therefore be neglected at large $N_{c}$, and we will not try to include it and analogous contributions for higher moments in the generating function. The physics of this contribution was discussed in Ref. [24]. There, it was shown that it corresponds to the Bose enhancement of gluons in the target wave function. Note that in the framework of the dilute target expansion utilized in Ref. [13] this contribution is leading order in $N_{c}$. The different $N_{c}$ counting for the same quantity in the dense and dilute limits is not uncommon in saturation approaches.

\section{B. Quadrupole contributions}

We now concentrate on the other two contractions of the projectile color charges. There are two such contractions, and they both lead to a single trace "quadrupole" contribution to the production probability:

$$
\begin{aligned}
& \overbrace{\left\langle\rho_{\mathrm{p}}^{a}\left(-\underline{q}^{\prime}\right)\left[U^{\dagger}\left(\underline{k}_{1}-\underline{q}^{\prime}\right) U\left(\underline{k}_{1}-\underline{q}\right)\right]_{a b} \rho_{\mathrm{p}}^{b}(\underline{q}) \rho_{\mathrm{p}}^{c}\left(-\underline{p}^{\prime}\right)\left[U^{\dagger}\left(\underline{k}_{2}-\underline{p}^{\prime}\right) U\left(\underline{k}_{2}-\underline{p}\right)\right]_{c d} \rho_{\mathrm{p}}^{d}(\underline{p})\right\rangle_{\mathrm{p}}}^{d N / d^{2} k_{1} d y_{1}} \\
& -\overbrace{\left\langle\rho_{\mathrm{p}}^{a}\left(-\underline{q}^{\prime}\right)\left[U^{\dagger}\left(\underline{k}_{1}-\underline{q}^{\prime}\right) U\left(\underline{k}_{1}-\underline{q}\right)\right]_{a b} \rho_{\mathrm{p}}^{b}(\underline{q})\right\rangle_{\mathrm{p}}}^{d N / d^{2} k_{1} d y_{1}} \overbrace{\left\langle\rho_{\mathrm{p}}^{c}\left(-\underline{p}^{\prime}\right)\left[U^{\dagger}\left(\underline{k}_{2}-\underline{p}^{\prime}\right) U\left(\underline{k}_{2}-\underline{p}\right)\right]_{c d} \rho_{\mathrm{p}}^{d}(\underline{p})\right\rangle_{\mathrm{p}}}^{d N / d^{2} k_{2} d y_{2}} \\
& =(2 \pi)^{2} \mu_{\mathrm{p}}^{2}(\underline{p}) \delta\left(\underline{p}-\underline{q}^{\prime}\right)(2 \pi)^{2} \mu_{\mathrm{p}}^{2}(\underline{q}) \delta\left(\underline{q}-\underline{p}^{\prime}\right) \operatorname{tr}\left[U^{\dagger}\left(\underline{k}_{1}-\underline{p}\right) U\left(\underline{k}_{1}-\underline{q}\right) U^{\dagger}\left(\underline{k}_{2}-\underline{q}\right) U\left(\underline{k}_{2}-\underline{p}\right)\right] \\
& +(2 \pi)^{2} \mu_{\mathrm{p}}^{2}\left(-\underline{p}^{\prime}\right) \delta\left(-\underline{p}^{\prime}-\underline{q}^{\prime}\right)(2 \pi)^{2} \mu_{\mathrm{p}}^{2}(\underline{q}) \delta(\underline{q}+\underline{p}) \operatorname{tr}\left[U^{\dagger}\left(\underline{k}_{1}-\underline{q}^{\prime}\right) U\left(\underline{k}_{1}-\underline{q}\right) U^{\dagger}\left(-\underline{q}-\underline{k}_{2}\right) U\left(-\underline{q}^{\prime}-\underline{k}_{2}\right)\right] \text {. }
\end{aligned}
$$

Each term has two contractions with respect to $U$ of order $N_{c}^{2}$. We organize them according to their physical meaning [24].

\section{HBT term}

The following contraction leads to the HBT contribution (cyclic property of trace was used):

$$
\begin{aligned}
& (2 \pi)^{4} \mu_{\mathrm{p}}^{2}(\underline{p}) \mu_{\mathrm{p}}^{2}(\underline{q}) \delta\left(\underline{p}-\underline{q}^{\prime}\right) \delta\left(\underline{q}-\underline{p}^{\prime}\right) \operatorname{tr}\left[\left\langle U\left(\underline{k}_{2}-\underline{p}\right) U^{\dagger}\left(\underline{k}_{1}-\underline{q}^{\prime}\right)\right\rangle_{\mathrm{t}}\left\langle U\left(\underline{k}_{1}-\underline{q}^{\prime} U^{\dagger}\left(\underline{k}_{2}-\underline{p}^{\prime}\right)\right\rangle_{\mathrm{t}}\right]\right. \\
& \quad+(2 \pi)^{4} \mu_{\mathrm{p}}^{2}\left(-\underline{p}^{\prime}\right) \mu_{\mathrm{p}}^{2}(\underline{q}) \delta\left(-\underline{p}^{\prime}-\underline{q}^{\prime}\right) \delta(\underline{q}+\underline{p}) \operatorname{tr}\left[\left\langle U\left(\underline{p}^{\prime}-\underline{k}_{2}\right) U^{\dagger}\left(\underline{k}_{1}-\underline{q}^{\prime}\right)\right\rangle_{\mathrm{t}}\left\langle U\left(\underline{k}_{1}-\underline{q}\right) U^{\dagger}\left(\underline{p}-\underline{k}_{2}\right)\right\rangle_{\mathrm{t}}\right] \\
& =\left(N_{c}^{2}-1\right)(2 \pi)^{8} S_{\perp}\left[\mu_{\mathrm{p}}^{2}(\underline{p}) \mu_{\mathrm{p}}^{2}(\underline{q}) D\left(\underline{k}_{1}-\underline{p}\right) D\left(\underline{k}_{1}-\underline{q}\right) \delta\left(\underline{k}_{2}-\underline{k}_{1}\right)+\mu_{\mathrm{p}}^{2}\left(-\underline{p}^{\prime}\right) \mu_{\mathrm{p}}^{2}(\underline{q}) D\left(\underline{p}^{\prime}+\underline{k}_{1}\right) D\left(\underline{k}_{1}-\underline{q}\right) \delta\left(\underline{k}_{1}+\underline{k}_{2}\right)\right] .
\end{aligned}
$$

Substituting into double inclusive production, we get

$$
\left[\frac{d^{2} N}{d y_{1} d y_{2}}\right]_{\mathrm{HBT}}=2\left(N_{c}^{2}-1\right) S_{\perp}\left(\frac{2 g^{2}}{(2 \pi)^{3}}\right)^{2} \int_{k_{\min }} d^{2} k \int d^{2} q d^{2} p \Gamma(\underline{k}, \underline{q}, \underline{p}) \Gamma(\underline{k}, \underline{p}, \underline{q}) \mu_{\mathrm{p}}^{2}(\underline{q}) \mu_{\mathrm{p}}^{2}(\underline{p}) D(\underline{k}-\underline{q}) D(\underline{k}-\underline{p}) .
$$

\section{Bose enhancement in the projectile}

The remaining contraction reflects Bose enhancement of gluons in the projectile wave function:

$$
\begin{aligned}
& (2 \pi)^{4} \mu_{\mathrm{p}}^{2}(\underline{p}) \mu_{\mathrm{p}}^{2}(\underline{q}) \delta\left(\underline{p}-\underline{q}^{\prime}\right) \delta\left(\underline{q}-\underline{p}^{\prime}\right) \operatorname{tr}\left[\left\langle U^{\dagger}\left(\underline{k}_{1}-\underline{q}^{\prime}\right) U\left(\underline{k}_{1}-q\right)\right\rangle_{\mathrm{t}}\left\langle U^{\dagger}\left(\underline{k}_{2}-\underline{p}^{\prime}\right) U\left(\underline{k}_{2}-\underline{p}\right)\right\rangle_{\mathrm{t}}\right] \\
& +(2 \pi)^{4} \mu_{\mathrm{p}}^{2}\left(-\underline{p}^{\prime}\right) \mu_{\mathrm{p}}^{2}(\underline{q}) \delta\left(-\underline{p}^{\prime}-\underline{q}^{\prime}\right) \delta(\underline{q}+\underline{p}) \operatorname{tr}\left[\left\langle U^{\dagger}\left(\underline{k}_{1}-\underline{q}^{\prime}\right) U\left(\underline{k}_{1}-\underline{q}\right)\right\rangle_{\mathrm{t}}\left\langle U^{\dagger}\left(\underline{p}-\underline{k}_{2}\right) U\left(\underline{p}^{\prime}-\underline{k}_{2}\right)\right\rangle_{\mathrm{t}}\right] \\
& =(2 \pi)^{8}\left(N_{c}^{2}-1\right) S_{\perp}\left[\mu_{\mathrm{p}}^{2}(\underline{p}) \mu_{\mathrm{p}}^{2}(\underline{q}) D\left(\underline{k}_{1}-\underline{q}\right) D\left(\underline{k}_{2}-\underline{q}\right) \delta\left(\underline{q^{\prime}}-\underline{q}\right) \delta\left(\underline{p}^{\prime}-\underline{p}\right) \delta(\underline{p}-\underline{q})\right. \\
& \left.\quad+\mu_{\mathrm{p}}^{2}(-\underline{p}) \mu_{\mathrm{p}}^{2}(\underline{q}) D\left(\underline{k}_{1}-\underline{q}\right) D\left(\underline{p}-\underline{k}_{2}\right) \delta\left(\underline{q}^{\prime}-\underline{q}\right) \delta\left(\underline{p^{\prime}}-\underline{p}\right) \delta(\underline{p}+\underline{q})\right] .
\end{aligned}
$$


Again, substituting into double inclusive production,

$$
\left[\frac{d^{2} N}{d y_{1} d y_{2}}\right]_{\mathrm{BE}}=2\left(N_{c}^{2}-1\right) S_{\perp} \int d^{2} q\left|\mu_{\mathrm{p}}^{2}(\underline{q})\right|^{2}\left|\frac{2 g^{2}}{(2 \pi)^{3}} \int_{k_{\min }} d^{2} k \Gamma(\underline{k}, \underline{q}, \underline{q}) D(\underline{q}-\underline{k})\right|^{2} .
$$

\section{Conclusions on double inclusive}

Comparing the HBT term Eq. (24) and the BE term Eq. (26), we see immediately that Eq. (26) gives the leading contribution to the multiplicity fluctuations.

The dominant contribution to the integral over $q$ in Eq. (26) comes from small $q$ due to the IR divergence of the Lipatov vertex

$$
\Gamma(\underline{k}, \underline{q}, \underline{q})=\frac{(\underline{k}-\underline{q})^{2}}{k^{2} q^{2}} .
$$

Thus, approximately, we have

$$
\begin{aligned}
& {\left[\frac{d^{2} N}{d y_{1} d y_{2}}\right]_{\mathrm{BE}}} \\
& \approx 2\left(N_{c}^{2}-1\right) S_{\perp} \int d^{2} q \frac{\left|\mu_{\mathrm{p}}^{2}(\underline{q})\right|^{2}}{q^{4}}\left|\frac{2 g^{2}}{(2 \pi)^{3}} \int_{k_{\min }} d^{2} k D(\underline{k})\right|^{2} .
\end{aligned}
$$

For the MV model, $\mu_{\mathrm{p}}^{2}=$ const, we get a strong IR divergence of the integral $\int d^{2} q \frac{\mu_{\mathrm{p}}^{4}}{q^{4}}$. This divergence is regularized by the momentum scale inversely proportional to the projectile size $\Lambda=\frac{1}{R_{\mathrm{p}}}$, that is, modulo constant factors

$$
\int d^{2} q \frac{\mu_{\mathrm{p}}^{4}}{q^{4}} \propto \mu_{\mathrm{p}}^{4} S_{\perp}
$$

where $S_{\perp}$ is the area of the projectile. Thus, the area dependence of the $\mathrm{BE}$ contribution to the double inclusive cross section is the same as that of the single inclusive cross section squared. This feature was noted and discussed earlier in Refs. [13,18], see also Ref. [25], in which the first saturation correction in the projectile to the double inclusive production was derived.

The behavior of the HBT contribution is different. There is no quadratic IR divergence for the integration over $q$ or $p$ in Eq. (24). As long as $k_{\min } \gg \Lambda$, no extra factor of area arises, and thus the HBT term is subleading. We note that if we include the soft scales in the $k$ integral, that is, take $k_{\min } \sim \Lambda$, the situation changes, and the HBT effect becomes as important as the Bose enhancement. We will not consider this situation in the present work.
Although we have concentrated on the double inclusive cross section, it is easy to see that the analysis generalizes to the higher gluon production as well [13]. For production of $n$ gluons, the BE term that has all contractions of the Wilson loops within the same single inclusive operator yields the highest power of area $S_{\perp}^{n-1}$. Thus, in the leading approximation, we will only keep these terms. We will discuss the first corrections to this approximation later on.

\section{MOMENT GENERATING FUNCTION-THE BOSE ENHANCEMENT CONTRIBUTION}

\section{A. Leading contribution from BE}

We now return to Eq. (16). We do not know how to perform the integration over $U$ in full generality. However, in view of the conclusion of the previous section, we will first only keep the leading BE contributions. It is quite clear how to do that. The leading $\mathrm{BE}$ contribution corresponds to contracting the two Wilson lines within the same single inclusive gluon operator. In the context of Eq. (16), this corresponds simply to contractions between the two Wilson lines inside the trace of the logarithm in the exponent,

$$
\begin{aligned}
G_{\mathrm{LO}}(t) & =\exp \left[-\frac{1}{2} \operatorname{tr} \ln \left[1-t\langle M\rangle_{t}\right]\right] \\
& =\exp \left[-\frac{1}{2}\left(N_{c}^{2}-1\right) S_{\perp} \int_{\Lambda}^{k_{\min }} \frac{d^{2} q}{(2 \pi)^{2}} \ln \left(1-t \frac{\mu_{\mathrm{p}}^{2}(q) \mathfrak{D}}{q^{2}}\right)\right],
\end{aligned}
$$

where we have defined the integrated dipole operator:

$$
\mathfrak{D}=\frac{4 g^{2}}{(2 \pi)^{3}} \int_{k_{\text {min }}} d^{2} k D(\underline{k}) \text {. }
$$

In Eq. (30), we have approximated the square of the Lipatov vertex by its leading term at low $q, \Gamma(k, q, q) \approx$ $1 / q^{2}$, and have restricted the integration over $q$ by $k_{\min }$ from above. These restrictions can be in principle lifted, but as long as $k_{\min } \gg \Lambda$, Eq. (30) faithfully represents all leading area terms in any moment of the probability distribution.

For the MV model $\mu_{\mathrm{p}}^{2}=$ const, the integral can be performed analytically, as follows: 


$$
\begin{aligned}
G_{\mathrm{LO}}(t) & =\exp \left[-\frac{1}{8 \pi}\left(N_{c}^{2}-1\right) S_{\perp} \int_{\Lambda^{2}}^{k_{\min }^{2}} d q^{2} \ln \left(1-t \frac{\mu_{\mathrm{p}}^{2} \mathfrak{D}}{q^{2}}\right)\right] \\
& =\exp \left[-\frac{1}{8 \pi}\left(N_{c}^{2}-1\right) S_{\perp}\left(k_{\min }^{2} \ln \left\{1-t \frac{\mu^{2} \mathfrak{D}}{k_{\min }^{2}}\right\}-\Lambda^{2} \ln \left\{1-t \frac{\mu^{2} \mathfrak{D}}{\Lambda^{2}}\right\}-t \mu^{2} \mathfrak{D} \ln \frac{k_{\min }^{2}-t \mu^{2} \mathfrak{D}}{\Lambda^{2}-t \mu^{2} \mathfrak{D}}\right)\right] \\
& =\exp \left[\frac{1}{8 \pi}\left(N_{c}^{2}-1\right) S_{\perp} \mu_{\mathrm{p}}^{2} \mathfrak{D}\left\{t \ln \frac{k_{\min }^{2}}{\Lambda^{2}}+\sum_{n=2}^{\infty} \frac{t^{n}}{n(n-1)}\left(\left(\frac{\mu_{\mathrm{p}}^{2} \mathfrak{D}}{\Lambda^{2}}\right)^{n-1}-\left(\frac{\mu_{\mathrm{p}}^{2} \mathfrak{D}}{k_{\min }^{2}}\right)^{n-1}\right)\right\}\right]
\end{aligned}
$$

The generating function for the cumulants is

$$
\begin{aligned}
\ln G_{\mathrm{LO}}(t) & =\frac{1}{8 \pi}\left(N_{c}^{2}-1\right) S_{\perp} \mu_{\mathrm{p}}^{2} \mathfrak{D}\left[\ln \frac{k_{\min }^{2}}{\Lambda^{2}} t+\sum_{n=2}^{\infty} \frac{1}{n(n-1)}\left\{\left(\frac{\mu_{\mathrm{p}}^{2} \mathfrak{D}}{\Lambda^{2}}\right)^{n-1}-\left(\frac{\mu_{\mathrm{p}}^{2} \mathfrak{D}}{k_{\min }^{2}}\right)^{n-1}\right\} t^{n}\right] \\
& \approx \frac{1}{8 \pi}\left(N_{c}^{2}-1\right) S_{\perp} \mu_{\mathrm{p}}^{2} \mathfrak{D}\left[\ln \frac{k_{\min }^{2}}{\Lambda^{2}} t+\sum_{n=2}^{\infty} \frac{1}{n(n-1)}\left(\frac{\mu_{\mathrm{p}}^{2} \mathfrak{D}}{\Lambda^{2}}\right)^{n-1} t^{n}\right] .
\end{aligned}
$$

This is our result for the multiplicity moment generating function, which resums all contributions due to the Bose enhancement of gluons in the projectile wave function. We now note some of its properties.

\section{B. Some properties of the distribution}

First of all, the mean number of particles, being the first moment of the distribution, is given by the first derivative of $G(t)$ at $t=0$ and is proportional to the projectile $\mu_{\mathrm{p}}^{2}$ and the logarithm of ratio of the momentum scales as expected,

$$
\kappa_{1}=\frac{1}{8 \pi}\left(N_{c}^{2}-1\right) S_{\perp} \mu_{\mathrm{p}}^{2} \mathfrak{D} \ln \frac{k_{\min }^{2}}{\Lambda^{2}} .
$$

The higher-order cumulants are given by

$\kappa_{n \geq 2}=\left.\frac{\partial}{\partial t^{n}} \ln G_{\mathrm{LO}}(t)\right|_{t=0}=(n-2) ! \frac{\left(N_{c}^{2}-1\right) S_{\perp} \Lambda^{2}}{8 \pi}\left(\frac{\mu_{\mathrm{p}}^{2} \mathfrak{D}}{\Lambda^{2}}\right)^{n}$.

Also note that, owing to presence of the increasing powers of $1 / \Lambda^{2}, \kappa_{n+1} \gg \kappa_{n}$, and thus the factorial cumulants defined by

$$
c_{n>2}=\left.\frac{\partial}{\partial z^{n}} \ln G_{\mathrm{LO}}(t=\ln z)\right|_{z=1}
$$

are approximately equal to the cumulants, $c_{n} \approx \kappa_{n}$.

The cumulants are very close to those of the $\gamma$ distribution,

$$
\bar{x} P(z=x / \bar{x})=\frac{\alpha}{\Gamma(\alpha)} e^{-z \alpha}(\alpha z)^{\alpha-1},
$$

with

$$
\kappa_{n}=(n-1) ! \alpha\left(\frac{\bar{x}}{\alpha}\right)^{n}
$$

Since the $\gamma$ distribution is known to exhibit the KobaNielsen-Olesen (KNO) scaling, we naturally expect to have KNO scaling at this order as well. We can check the KNO scaling by plotting the scaling function (37) for different values of $k_{\min }$. The value of $k_{\min }$ defines the mean multiplicity. This is plotted in Fig. 1, right panel. Note that what is plotted here is the result of full numerical evaluation of the probability distribution (see the Appendix for detail) and not just the leading approximation. The numerics is performed by using the MV model also for the target fields with the scale $\mu_{\mathrm{t}} \gg \mu_{\mathrm{p}}$. One observes that, indeed, for large enough $k_{\min }$, the quality of the KNO scaling is very good. There is one subtlety here. Our distribution (32) is not exactly the $\gamma$ distribution. In particular, the first moment (34) has an additional logarithmic factor relative to the parameter that determines the higher moments (35). Since this logarithmic factor depends on $k_{\min }$, it could potentially affect the KNO scaling. Nevertheless, this additional logarithmic dependence is very slowly varying, and the scaling is clearly seen in the numerical results in Fig. 1.

The left panel in Fig. 1 illustrates what happens when the soft scale $\Lambda$ is raised and becomes comparable with the target saturation momentum. One clearly observes that as $\Lambda$ grows the probability distribution becomes narrower. Note that in this regime we cannot neglect the effects of HBT as well as correction due to the exact form of the Lipatov vertex. These are the effects that drive the narrowing of the distribution for larger $\Lambda$.

The leading-order probability distribution we obtained is similar in many respects to that obtained in Ref. [13]. There are, however, some significant differences. First, in the case of the dense target, the contribution of the Bose enhancement in the target wave function is suppressed by the factor $1 / N_{c}^{2}$, whereas in Ref. [13], the target was treated as dilute; this contribution was of order unity and contributed to the probability distribution on par with the projectile $\mathrm{BE}$. 

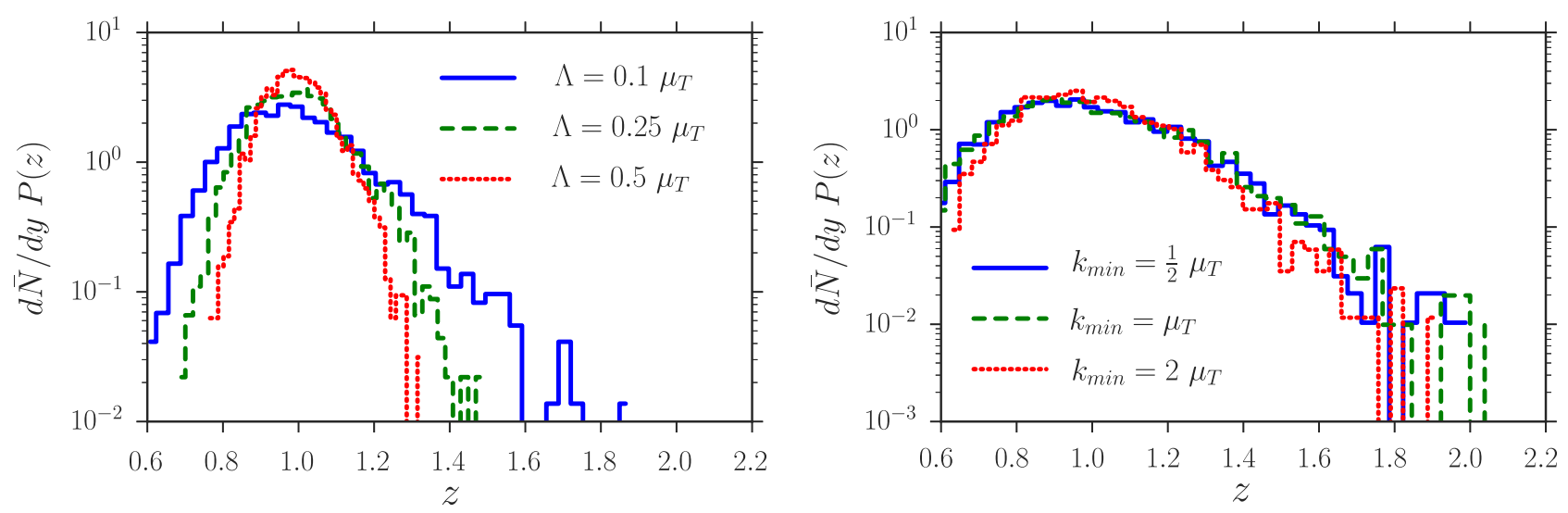

FIG. 1. The probability distribution multiplied by the average as a function of $z=d N / d y /(d \bar{N} / d y)$ for different values of the infrared cut-off, $\Lambda$, and the minimal momentum of produced gluon, $k_{\min }$.

Second, the cumulants in our case are very close to those given by the gamma distribution, whereas Ref. [13] found negative binomial distribution (NBD). The $n$th cumulants of the two distributions differ by the factor $n-1$. This difference can be traced to the different ways the function $\mu_{\mathrm{p}}^{2}(p)$ is treated at small $p$ in the two approaches. Our calculation corresponds to taking constant $\mu_{\mathrm{p}}^{2}$ and cutting off the putative infrared divergence in the integrals by a finite area of the projectile. The IR regulator therefore does not arise from making the correlation between the sources nonlocal in coordinate space but rather imposing an impact parameter profile on the source density. On the other hand, the authors of Ref. [13] regulated IR divergences by taking $\mu_{\mathrm{p}}^{2}(p) \sim p^{2}$ for momenta smaller than the projectile saturation momentum. Physically, this corresponds to taking the correlation function between the two sources to be nonlocal in coordinate space.

This different treatment of the IR behavior leads to different integrals with respect to the incoming gluon from the projectile wave function $q$. In this paper, we keep $\mu_{\mathrm{p}}=$ const and thus

$$
\int d q^{2} \frac{\mu_{\mathrm{p}}^{2 n}}{q^{2 n}}=-\frac{1}{n-1} \frac{\mu_{\mathrm{p}}^{2 n}}{q^{2(n-1)}},
$$

while the authors of Ref. [13] approximated $\mu_{\mathrm{p}}^{2} \approx q^{2}$; in this case, the integral in Eq. (39) brings no additional factors dependent on $n$.

Related to this point is also the different energy dependence that we expect from the distribution. In our case, the parameter of the distribution is $\alpha=\frac{N_{c}^{2}-1}{8 \pi} S_{\perp} \Lambda^{2}$, where $S_{\perp} \Lambda^{2} \approx 1$. This parameter does not depend on energy for the dilute projectile. Thus, we expect $\alpha$ to be approximately energy independent. On the other hand, the treatment of the IR in Ref. [13] leads to the replacement of $\Lambda^{2}$ by the saturation momentum of the projectile $Q_{s}^{2}$. This grows with energy, and thus the probability distribution has rather strong dependence on energy.

\section{Constraint action formalism and the Liouville potential}

One interesting property of our derivation is that the probability distribution of produced particles that we find is deeply related to the probability distribution of particles in the projectile wave function. Note that we have approximated the Lipatov vertex by its part that involves only the momentum of the gluon coming from the incoming wave function. Thus, if in the rest of the calculation we simply set $U(\underline{x})=1$, that would correspond to calculating the particle distribution in the projectile wave function. This last move would simply correspond to setting $k_{\min }=0$ in Eq. (31), since $\int \frac{d^{2} k}{(2 \pi)^{2}} D(\underline{k})=D(\underline{x}=0)=1$, which is equivalent to setting $U=1$. This then gives

$$
\mathfrak{D}=\frac{2 g^{2}}{\pi}
$$

Clearly, we should not set $k_{\min }=0$ in the integration limits in Eq. (30) but instead keep it fixed. Thus, we conclude that taking the limit of Eq. (40) in Eq. (30) corresponds to calculating the probability distribution for particles with transverse momenta smaller than momentum $k_{\min }$ in the projectile wave function.

Interestingly, such a calculation can be performed independently using an alternative formulation based on the framework of the constraint effective potential; see Refs. [19,26,27].

The main idea of this approach is to integrate out fluctuations of $\rho_{\mathrm{p}}(\underline{q})$ that do not affect a specific operator defined through $\rho_{\mathrm{p}}(\underline{q})$. In Ref. [19], the constraint effective potential for the gluon distribution defined by the covariant field, $A^{+}$, was derived,

$$
e^{-V_{\text {eff }}[\eta(\underline{q})]}=\frac{1}{Z_{\mathrm{p}}} \int \mathcal{D} \rho_{\mathrm{p}} W\left(\rho_{\mathrm{p}}\right) \delta\left(\eta(\underline{q})-\frac{g^{2} \operatorname{tr}\left|A^{+}(\underline{q})\right|^{2}}{\left\langle g^{2} \operatorname{tr}\left|A^{+}(\underline{q})\right|^{2}\right\rangle}\right),
$$


where $A^{+}(\underline{q})=g / q^{2} \rho_{\mathrm{p}}(\underline{q})$,

$$
\left\langle g^{2} \operatorname{tr}\left|A^{+}(\underline{q})\right|^{2}\right\rangle=\frac{1}{2}\left(N_{c}^{2}-1\right) S_{\perp} \frac{g^{4} \mu_{\mathrm{p}}^{2}}{q^{4}}
$$

and

$V_{\mathrm{eff}}[\eta(\underline{q})]=\frac{1}{2}\left(N_{c}^{2}-1\right) S_{\perp} \int \frac{d^{2} q}{(2 \pi)^{2}}\{\eta(\underline{q})-1-\ln \eta(\underline{q})\}$.
This potential corresponds to a Liouville potential with negative Ricci scalar. ${ }^{2}$

The probability distribution for gluon multiplicity in the projectile is defined as

$G_{\mathrm{LO}}(t)=\left\langle\exp \left[t \int_{\Lambda}^{k_{\min }} \frac{d^{2} q}{(2 \pi)^{2}} \rho_{\mathrm{p}}^{a}(-\underline{q}) \frac{\mathfrak{D}}{2 q^{2}} \rho_{\mathrm{p}}^{a}(\underline{q})\right]\right\rangle_{\mathrm{p}}$.

Using the effective potential (43), we have

$$
\begin{aligned}
G_{\mathrm{LO}}(t) & =\left\langle\exp \left[t \int_{\Lambda}^{k_{\min }} \frac{d^{2} q}{(2 \pi)^{2}} \rho_{\mathrm{p}}^{a}(-\underline{q}) \frac{\mathfrak{D}}{2 q^{2}} \rho_{\mathrm{p}}^{a}(\underline{q})\right]\right\rangle_{\mathrm{p}} \\
& =\int \mathcal{D} \eta \exp \left(-V_{\mathrm{eff}}[\eta(\underline{q})]+\frac{1}{2}\left(N_{c}^{2}-1\right) S_{\perp} \int_{\Lambda}^{k_{\min }} \frac{d^{2} q}{(2 \pi)^{2}} t \frac{\mu_{\mathrm{p}}^{2} \mathfrak{D}}{q^{2}} \eta(\underline{q})\right) \\
& =\int \mathcal{D} \eta \exp \left(-\frac{1}{2}\left(N_{c}^{2}-1\right) S_{\perp} \int \frac{d^{2} q}{(2 \pi)^{2}}\left\{\eta(\underline{q})-1-\ln \eta(\underline{q})+\theta(q-\Lambda) \theta\left(k_{\min }-q\right) t \frac{\mu_{\mathrm{p}}^{2} \mathfrak{D}}{q^{2}} \eta(\underline{q})\right\}\right) .
\end{aligned}
$$

For large $S_{\perp}$, this integral can be computed using the saddle point approximation

$\eta_{\mathrm{SP}}(\underline{q})=\left\{\begin{array}{l}\left(1-t \frac{\mu_{\mathrm{p}}^{2} \mathfrak{D}}{q^{2}}\right)^{-1}, \quad \text { if } \Lambda \leq q \leq k_{\mathrm{min}} \\ 1, \text { otherwise }\end{array}\right.$

to yield

$$
\begin{aligned}
G_{\mathrm{LO}}(t) & =\exp \left[\frac{1}{2}\left(N_{c}^{2}-1\right) S_{\perp} \int \frac{d^{2} q}{(2 \pi)^{2}} \ln \eta_{\mathrm{SP}}(\underline{q})\right] \\
& =\exp \left[-\frac{1}{2}\left(N_{c}^{2}-1\right) S_{\perp} \int_{\Lambda}^{k_{\min }} \frac{d^{2} q}{(2 \pi)^{2}} \ln \left(1-t \frac{\mu_{\mathrm{p}}^{2} \mathfrak{D}}{q^{2}}\right)\right],
\end{aligned}
$$

which reproduces the result obtained previously. Interestingly, the origin of the logarithm in this equation is owing to the presence of the Liouville logarithm in the effective constraint action for $\eta(\underline{q})$; see Eq. (43).

Note that the form of the integral appearing in Eq. (45) is quite suggestive,

$$
\begin{aligned}
& \int \mathcal{D} \eta \exp \left(-V_{\mathrm{eff}}[\eta(\underline{q})]+t \frac{1}{2}\left(N_{c}^{2}-1\right) S_{\perp}\right. \\
&\left.\times \int_{\Lambda}^{k_{\min }} \frac{d^{2} q}{(2 \pi)^{2}} \frac{\mu_{\mathrm{p}}^{2} \mathfrak{D}}{q^{2}} \eta(\underline{q})\right) .
\end{aligned}
$$

\footnotetext{
${ }^{2}$ See also Ref. [28], in which this formalism was applied to describe the centrality dependence of the nuclear modification factor.
}

Here, $t$ can be viewed as an external field, while the moments and the cumulants of the gluon number can be viewed as the moments and the cumulants for fluctuations of the composite field $\int d^{2} q \eta(q) / q^{2}$. Thus, the fluctuations of the number of particles provide a direct measurement of the Liouville potential.

It is interesting to note that our derivation provides a concrete realization of early ideas in the literature about relevance of Liouville action to multiplicity fluctuations in CGC. Reference [29] postulated ad hoc such a Liouville potential for saturation momentum fluctuations. In the present paper, we instead derive it form the constrained effective potential for the MV model. ${ }^{3}$

Although the argument of our potential is not the saturation momentum, but rather the composite filed $\eta$, they are closely related. Consider the effective potential for $\eta$ close to its saddle point value at zero external field. Expanding in $\ln \eta$, we obtain

$$
V_{\mathrm{eff}}[\eta(\underline{q})] \approx \frac{1}{2}\left(N_{c}^{2}-1\right) S_{\perp} \int \frac{d^{2} q}{(2 \pi)^{2}} \frac{1}{2} \ln ^{2} \eta(\underline{q}) .
$$

Recall that the operator definition of the composite field $\eta$ is given by Eq. (41),

$$
\eta(\underline{q})=\frac{\rho_{\mathrm{p}}^{a}(\underline{q}) \rho_{\mathrm{p}}^{a}(\underline{q})}{\left(N_{c}^{2}-1\right) \mu_{\mathrm{p}}^{2}(\underline{q})},
$$

\footnotetext{
${ }^{3}$ Although our derivation was done in the MV model, the numerical result of Ref. [19], see Fig. 5, suggests that the highenergy evolution does not change the form of the potential and only leads to the modification of the effective projectile area $S_{\perp}$. This modification might be responsible for the origin of the effective width $\sigma$ used in Refs. [30,31].
} 
which is interpreted naturally as the scaled fluctuating saturation momentum of the projectile, since on a configuration by configuration basis the saturation momentum is indeed determined by the square of the color charge density. Thus, we indeed can interpret Eq. (49) as the probability distribution for the fluctuating saturation momentum, which has exactly the same form as assumed in Refs. [30,31].

\section{CORRECTIONS TO THE GENERATING FUNCTION}

In this section, we consider corrections to the cumulant generating function. There are two basic types of corrections: those attributed to $\mathrm{BE}$ with a lower power of IR divergence when integrating with respect to the incoming gluon from the projectile wave function and those attributed to the HBT contribution; see the section for two-particle gluon production.

\section{A. Subleading BE terms}

The subleading IR terms due to Bose enhancement can be easily resummed. We simply have to allow for the full
Lipatov vertex and for the $q$ dependence of the dipole amplitude in Eq. (30). Thus, formally, we get

$$
\begin{aligned}
G_{\mathrm{BE}}(t)= & \exp \left[-\frac{1}{2}\left(N_{c}^{2}-1\right) S_{\perp}\right. \\
& \left.\times \int_{\Lambda}^{k_{\min }} \frac{d^{2} q}{(2 \pi)^{2}} \ln \left(1-t \mu_{\mathrm{p}}^{2}(\underline{q}) \overline{\mathfrak{D}}(q)\right)\right],
\end{aligned}
$$

where

$$
\overline{\mathfrak{D}}(q)=\frac{4 g^{2}}{(2 \pi)^{3}} \int_{k_{\min }} d^{2} k D(\underline{k}-\underline{q}) \Gamma(\underline{k}, \underline{q}, \underline{q}) .
$$

The explicit form of the distribution now depends on the dipole amplitude $D(k)$ and can be calculated once this amplitude is known.

\section{B. HBT contributions}

We now concentrate on the corrections of the second type. We will keep only terms leading in $N_{c}$.

We rewrite our basic expression for the generating function as

$$
\begin{aligned}
G(t)= & \int \frac{\mathcal{D} \rho_{\mathrm{p}}}{Z_{\mathrm{p}}} \int \frac{\mathcal{D} \rho_{\mathrm{t}}}{Z_{\mathrm{t}}} W_{\mathrm{p}}\left(\rho_{\mathrm{p}}\right) W_{\mathrm{t}}\left(\rho_{\mathrm{t}}\right) \exp \left[t \frac{2 g^{2}}{(2 \pi)^{3}} \int \frac{d^{2} q}{(2 \pi)^{2}} \frac{d^{2} q^{\prime}}{(2 \pi)^{2}} \rho_{\mathrm{p}}^{a}\left(-\underline{q}^{\prime}\right) \int d^{2} k \Gamma\left(\underline{k}, \underline{q}, \underline{q^{\prime}}\right)\left[U^{\dagger}\left(\underline{k}-\underline{q}^{\prime}\right) U(\underline{k}-\underline{q})\right]_{a b} \rho_{\mathrm{p}}^{b}(\underline{q})\right] \\
= & \int \frac{\mathcal{D} \rho_{\mathrm{p}}}{Z_{\mathrm{p}}} W_{\mathrm{p}}\left(\rho_{\mathrm{p}}\right) \exp \left[t \frac{1}{2} \int \frac{d^{2} q}{(2 \pi)^{2}} \rho_{\mathrm{p}}^{a}(-\underline{q}) \bar{D}(q) \rho_{\mathrm{p}}^{a}(\underline{q})\right] \\
& \times \int \frac{\mathcal{D} \rho_{\mathrm{t}}}{Z_{\mathrm{t}}} W_{\mathrm{t}}\left(\rho_{\mathrm{t}}\right) \exp \left[t \frac{2 g^{2}}{(2 \pi)^{3}} \int \frac{d^{2} q}{(2 \pi)^{2}} \frac{d^{2} q^{\prime}}{(2 \pi)^{2}} \rho_{\mathrm{p}}^{a}\left(-\underline{q^{\prime}}\right)\left\{\int d^{2} k \Gamma\left(\underline{k}, \underline{q}, \underline{q^{\prime}}\right):\left[U^{\dagger}\left(\underline{k}-\underline{q}^{\prime}\right) U(\underline{k}-\underline{q})\right]_{a b}:\right\} \rho_{\mathrm{p}}^{b}(\underline{q})\right] .
\end{aligned}
$$

The BE contribution represented by the first line of Eq. (54) is discussed in the detail in previous section. The last two terms are the corrections we are after. We have introduced the normal-ordered product of the Wilson lines

$$
:\left[U^{\dagger}\left(\underline{k}-\underline{q}^{\prime}\right) U(\underline{k}-\underline{q})\right]_{a b}: \equiv\left[U^{\dagger}\left(\underline{k}-\underline{q}^{\prime}\right) U(\underline{k}-\underline{q})\right]_{a b}-D(\underline{k}-\underline{q}) \delta_{a b}(2 \pi)^{2} \delta\left(\underline{q}^{\prime}-\underline{q}\right) .
$$

The normal ordering ensures that, apart from the BE term, no other terms contain contractions between two Wilson lines that belong to the same "vertex." These contractions have been completely resummed into the propagator of the color charge density,

$$
\frac{1}{\hat{\mu}_{\mathrm{p}}^{2}(q)}=\frac{1}{\mu_{\mathrm{p}}^{2}}-t \overline{\mathfrak{D}}(q) \approx \frac{1}{\mu_{\mathrm{p}}^{2}}-t \frac{\mathfrak{D}}{q^{2}} .
$$

We can expand the functional integral into series in the "interaction term,"

$$
\begin{aligned}
G(t) & =\int \frac{\mathcal{D} \rho_{\mathrm{p}}}{Z_{\mathrm{p}}} \exp \left[-\int \frac{d^{2} q}{(2 \pi)^{2}} \rho_{\mathrm{p}}^{a}(-\underline{q}) \frac{1}{2 \hat{\mu}_{\mathrm{p}}^{2}} \rho_{\mathrm{p}}^{a}(\underline{q})\right] \int \frac{\mathcal{D} \rho_{\mathrm{t}}}{Z_{\mathrm{t}}} W_{\mathrm{t}}\left(\rho_{\mathrm{t}}\right) \exp \left[S_{\mathrm{int}}\left(\rho_{\mathrm{p}}, \rho_{\mathrm{t}}\right)\right] \\
& =\int \frac{\mathcal{D} \rho_{\mathrm{p}}}{Z_{\mathrm{p}}} \exp \left[-\int \frac{d^{2} q}{(2 \pi)^{2}} \rho_{\mathrm{p}}^{a}(-\underline{q}) \frac{1}{2 \hat{\mu}_{\mathrm{p}}^{2}} \rho_{\mathrm{p}}^{a}(\underline{q})\right] \sum_{n} \frac{1}{n !} \int \frac{\mathcal{D} \rho_{\mathrm{t}}}{Z_{\mathrm{t}}} W_{\mathrm{t}}\left(\rho_{\mathrm{t}}\right) S_{\text {int }}^{n}\left(\rho_{\mathrm{p}}, \rho_{\mathrm{t}}\right),
\end{aligned}
$$

where the interaction part is given by

$$
S_{\text {int }}\left(\rho_{\mathrm{p}}, \rho_{\mathrm{t}}\right)=t \frac{2 g^{2}}{(2 \pi)^{3}} \int \frac{d^{2} q}{(2 \pi)^{2}} \frac{d^{2} q^{\prime}}{(2 \pi)^{2}} \rho_{\mathrm{p}}^{a}\left(-\underline{q^{\prime}}\right)\left\{\int d^{2} k \Gamma\left(\underline{k}, \underline{q}, \underline{q^{\prime}}\right):\left[U^{\dagger}\left(\underline{k}-\underline{q^{\prime}}\right) U(\underline{k}-\underline{q})\right]_{a b}:\right\} \rho_{\mathrm{p}}^{b}(\underline{q}) .
$$


(a)

(b)

(c)

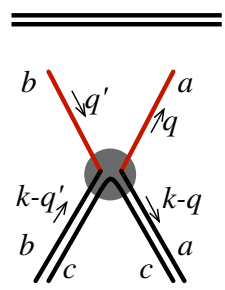

FIG. 2. Feynman rules for the propagators and the vertex: a) the resummed propagator of the projectile $\rho: \hat{\mu}_{\mathrm{p}}^{2}(\underline{q}) \delta_{a b} ;$ b) the propagator of the target $U: D(\underline{q}) /\left(N_{c}^{2}-1\right) \delta_{b c} \delta_{c d}$; c) the "interaction vertex": $t \frac{8 g^{2}}{(2 \pi)^{3}} \Gamma\left(\underline{k}, \underline{q}, \underline{q}^{\prime}\right)$.

To compute the cumulant generating function, it is useful to introduce the Feynman rules depicted in Fig. 2. Note that, due to normal ordering in the vertex, no diagram with contraction of the two U's belonging to the same vertex is allowed. Those have already been resummed in the propagator of $\rho_{\mathrm{p}}$.
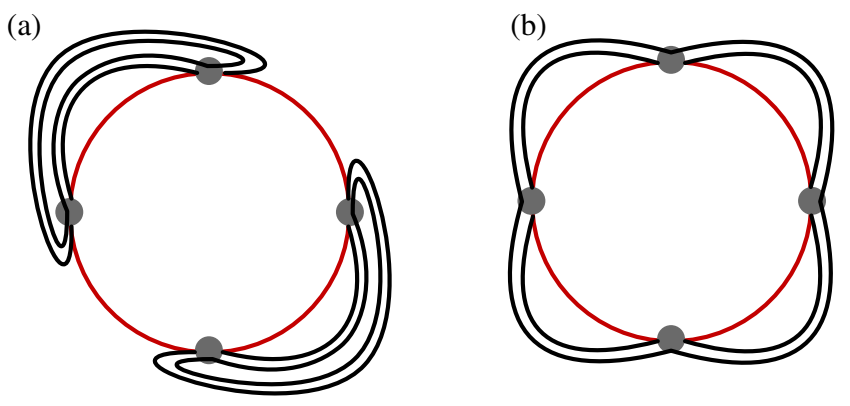

FIG. 3. Example of the leading $N_{c}$ contribution.

To understand which type of diagrams gives the leading corrections, let us first consider a particular example: the connected contribution involving four vertices (correction to inclusive four-particle production). The leading $N_{c}$ contributions have two different topologies; see Fig. 3.

Diagram a in Fig. 3 is given by (modulo combinatorial and kinematic factors)

$$
\int d^{2} k_{1} d^{2} k_{2} \int \frac{d^{2} q}{(2 \pi)^{2}} \frac{d^{2} q_{1}}{(2 \pi)^{2}} \frac{d^{2} q_{2}}{(2 \pi)^{2}} \Gamma^{2}\left(\underline{k}_{1}, \underline{q}, \underline{q}_{1}\right) \Gamma^{2}\left(\underline{k}_{2}, \underline{q}, \underline{q}_{2}\right) \hat{\mu}_{\mathrm{p}}^{4}(\underline{q}) \hat{\mu}_{\mathrm{p}}^{2}\left(\underline{q}_{1}\right) \hat{\mu}_{\mathrm{p}}^{2}\left(\underline{q}_{2}\right) D\left(\underline{k}_{1}-\underline{q}\right) D\left(\underline{k}_{1}-\underline{q}_{1}\right) D\left(\underline{k}_{2}-\underline{q}\right) D\left(\underline{k}_{2}-\underline{q}_{2}\right) .
$$

Diagram $b$ in Fig. 3 is given by

$$
\begin{aligned}
& \int d^{2} k \int \frac{d^{2} q_{1}}{(2 \pi)^{2}} \frac{d^{2} q_{2}}{(2 \pi)^{2}} \frac{d^{2} q_{3}}{(2 \pi)^{2}} \frac{d^{2} q_{4}}{(2 \pi)^{2}} \Gamma\left(\underline{k}, \underline{q}_{1}, \underline{q}_{2}\right) \Gamma\left(\underline{k}, \underline{q}_{2}, \underline{q}_{3}\right) \Gamma\left(\underline{k}, \underline{q}_{3}, \underline{q}_{4}\right) \Gamma\left(\underline{k}, \underline{q}_{4}, \underline{q}_{1}\right) \hat{\mu}_{\mathrm{p}}^{2}\left(\underline{q}_{1}\right) \hat{\mu}_{\mathrm{p}}^{2}\left(\underline{q}_{2}\right) \hat{\mu}_{\mathrm{p}}^{2}\left(\underline{q}_{3}\right) \hat{\mu}_{\mathrm{p}}^{2}\left(\underline{q}_{4}\right) \\
& \quad \times D\left(\underline{k}-\underline{q}_{1}\right) D\left(\underline{k}-\underline{q}_{2}\right) D\left(\underline{k}-\underline{q}_{3}\right) D\left(\underline{k}-\underline{q}_{4}\right) .
\end{aligned}
$$

As before, we consider the IR dominant contribution from the Lipatov vertices. For diagram b, we get

$$
\begin{gathered}
\Gamma\left(\underline{k}, \underline{q}_{1}, \underline{q}_{2}\right) \Gamma\left(\underline{k}, \underline{q}_{2}, \underline{q}_{3}\right) \Gamma\left(\underline{k}, \underline{q}_{3}, \underline{q}_{4}\right) \Gamma\left(\underline{k}, \underline{q}_{4}, \underline{q}_{1}\right) \\
\quad \approx \frac{\underline{q}_{1} \cdot \underline{q}_{2}}{q_{1}^{2} q_{2}^{2}} \frac{\underline{q}_{2} \cdot \underline{q}_{3}}{q_{2}^{2} \underline{q}_{3} \cdot \underline{q}_{4}} \underline{q}_{4} \cdot \underline{q}_{1} \\
q_{3}^{2} q_{4}^{2}
\end{gathered}
$$

which, after angular integration, has logarithmic divergence for each integral of the form $\int d q_{i}^{2} / q_{i}^{2}$. For diagram a, we get

$$
\Gamma^{2}\left(\underline{k}_{1}, \underline{q}, \underline{q}_{1}\right) \Gamma^{2}\left(\underline{k}_{2}, \underline{q}, \underline{q}_{2}\right) \approx\left(\frac{\underline{q} \cdot \underline{q} \underline{q}_{1}}{q^{2} q_{1}^{2}} \frac{\underline{q} \cdot \underline{q}_{2}}{q^{2} q_{2}^{2}}\right)^{2}
$$

and in this case, the integral with respect to $q$ has quadratic divergence in the IR. This divergence is of course regulated by $\Lambda^{2} \propto 1 / S_{\perp}$, which leads to the extra power of $S_{\perp}$.
It is now clear what the leading diagrams due to the HBT corrections that contribute most to the generating function are. Those are the diagrams that contain the maximal number of the $\rho_{\mathrm{p}}$ propagators at the same momentum $q$, since each such propagator is accompanied by a product of two Lipatov vertices containing the same momentum $q$, thereby leading to one extra power of area for each additional $\rho_{\mathrm{p}}$ propagator. These diagrams are of the type of Fig. 3(a), in which all the vertices are organized into pairs with two vertices of the pair connected by two propagators of the $U$ field and one propagator of $\rho_{\mathrm{p}}$. Physically, this corresponds to contributions to the $n$-gluon inclusive production, in which gluon pairs are emitted independently but the HBT correlations are present between two gluons in each pair.

We are now going to ignore diagrams of type $\mathrm{B}$ but resum all diagrams of type $\mathrm{A}$, corresponding to pairwise HBT correlations. 
For a diagram with $2 n$ vertices, we have the following combinatorial coefficient $\frac{1}{(2 n) !}$ from expanding the exponential; $(2 n-1)$ !!, the number of ways to organize the $2 n$ vertices into pairs; $2^{n}$, the number of contractions of $U$, two possibilities within each pair of vertices; and $2^{n}$, the number of contractions between two $\rho_{\mathrm{p}}$ 's within the pair of vertices. Although there are four possible of contractions in general, once a particular contraction of $U$ 's is chosen, only two contractions of $\rho_{\mathrm{p}}$ 's are leading order in $N_{c} ; \frac{(n-1) !}{2}$ ways of ordering the $n$ vertex pairs along a circle.

Thus, at the end of the day, the diagram with $2 n$ vertices that are contracted pairwise into the "daisy" is $2^{2 n-1} \frac{1}{n}$.

Resumming these terms, we obtain

$$
\begin{aligned}
& \ln G(t)-\ln G_{\mathrm{LO}}(t) \\
& \approx \frac{\left(N_{c}^{2}-1\right)}{2} S_{\perp} \int_{\Lambda}^{k_{\min }} \frac{d^{2} q}{(2 \pi)^{2}} \sum_{n=1}^{\infty} \frac{1}{n} \mathcal{Z}^{n}(\underline{q}) \hat{\mu}_{\mathrm{p}}^{2 n}(\underline{q}) \\
& =-\frac{\left(N_{c}^{2}-1\right) S_{\perp}}{2} \int_{\Lambda}^{k_{\min }} \frac{d^{2} q}{(2 \pi)^{2}} \ln \left[1-t^{2} \frac{\hat{\mu}_{\mathrm{p}}^{2}(\underline{q})}{q^{2}}\right]
\end{aligned}
$$

with

$$
\mathcal{Z}=\int_{\Lambda}^{k_{\min }} \frac{d^{2} q^{\prime}}{(2 \pi)^{2}} \mathfrak{D}_{2}\left(q^{\prime}\right) \frac{\hat{\mu}_{\mathrm{p}}^{2}\left(\underline{q}^{\prime}\right)}{q^{\prime 2}}
$$

and

$$
\mathfrak{D}_{2}(q) \equiv\left(\frac{g^{2}}{\pi^{3}}\right)^{2} \int d^{2} k D(k) D(k-q) .
$$

Finally, we obtain the cumulant generating function in the form

$$
\begin{aligned}
\ln G(t) & =-\frac{\left(N_{c}^{2}-1\right) S_{\perp}}{2} \int_{\Lambda}^{k_{\min }} \frac{d^{2} q}{(2 \pi)^{2}} \ln \left\{\mu_{\mathrm{p}}^{2}(\underline{q})\left[\frac{1}{\hat{\mu}_{\mathrm{p}}^{2}(\underline{q})}-t^{2} \frac{\mathcal{Z}}{q^{2}}\right]\right\} \\
& =\frac{\left(N_{c}^{2}-1\right) S_{\perp}}{2} \int_{\Lambda}^{k_{\min }} \frac{d^{2} q}{(2 \pi)^{2}} \ln \left\{1-t \frac{\mu_{\mathrm{p}}^{2}}{q^{2}}(\mathfrak{D}+t Z)\right\},
\end{aligned}
$$

which has the same form as Eq. (32); thus, the momentum integral can be performed analytically, leading to Eq. (33) with the substitution $\mathfrak{D} \rightarrow(\mathfrak{D}+t Z)$.

This is a rather simple expression, and one can analyze the effects of the correction given a model for the dipole amplitude $D(p)$. These corrections may be important in the regime in which $k_{\min }$ is not significantly greater than the soft scale $\Lambda$. Although we do not consider such a situation in the present paper, at high enough energy, the projectile wave function itself will acquire a saturation momentum scale $Q_{\mathrm{s}, \mathrm{p}}$ significantly larger than $\Lambda$. In this case, it is quite conceivable that in our expressions the soft scale $\Lambda$ will be replaced by this semisoft scale $Q_{\mathrm{s}, \mathrm{p}}$. In this case, it is perfectly sensible to consider $k_{\min }<Q_{\mathrm{s}, \mathrm{p}}$. The HBT contributions in this regime will become significant, and the relative significance of the BE and HBT contributions to the multiplicity fluctuations has to be reanalyzed. We will not attempt to do it in the present paper.

\section{CONCLUSIONS}

In this paper, we studied the multiplicity fluctuations in $p-A$ collisions within the framework of the dense-dilute CGC formalism using the MV model for the wave function of the proton. Our approach is similar in many aspects to that of Ref. [13]. There are, however, some significant differences, and our results are quite different as well. As opposed to Ref. [13], we treat the target as very dense, while on the projectile side, we do not assume any dynamical "correlation length" associated with the saturation momentum. We rather treat the IR physics of the proton wave function as genuinely nonperturbative, governed by a soft scale of the order of the inverse proton size. As a result, we obtain the probability distribution, which within a large range of energies is energy independent.

We identified two sources of multiplicity fluctuations: those due to the Bose enhancement of gluons in the proton wave function and the HBT effect in the initial stages of scattering. Interestingly, in the dense-dilute framework, the Bose enhancement in the nucleus wave function leads only to a $\left(N_{c}^{2}-1\right)^{-1}$-suppressed contribution to any cumulant of particle number and is thus a subleading effect. We demonstrated that as long as the low momentum structure of the proton wave function is dominated by the genuine soft scale (the "proton size") the dominant origin for the multiplicity fluctuations is the Bose enhancement.

We have calculated explicitly the moment generating function for the multiplicity distribution due to BE. The distribution we obtain is very close to the $\gamma$ distribution. Just like the $\gamma$ distribution, it satisfies the KNO scaling with very good precision. Interestingly, the leading term in the generating function for the multiplicity of produced particles is practically identical to the generating function for multiplicity distribution in the projectile wave function. This latter quantity can be calculated using the effective action approach as suggested in Ref. [19]. We have shown that this effective action is nothing but the Liouville action for the composite field, which can be thought of as fluctuating density (or saturation momentum).

The authors of Ref. [13] obtained the negative binomial distribution for the multiplicity. Our result, as mentioned above, is somewhat different, although for large moments, the NBD and the $\gamma$ distribution are quite similar. The main difference, as explained in the text, is in the physics of the scale that regulates the formal IR divergences. In the densedilute calculation performed in the present paper, this role is played by the soft scale of the proton radius and not by the semisoft scale of the projectile saturation momentum. If one assumes that the proton wave function itself is 
characterized by a finite correlation length, much smaller than the proton size, one would have to reanalyze to what extent the dominance of the BE persists. It may well happen that the HBT contributions become equally important and have to be included in the leading-order calculation. In fact, this is precisely what happens on the target side, where the presence of large saturation momentum strongly suppresses the BE effect, as we noted above. It is thus not clear to us that the approximation of $\mathrm{BE}$ dominance and finite saturation momentum of the projectile are mutually compatible.

We note that both NBD and $\gamma$ distribution have rather long tails for large values of produced multiplicity. It is very natural that these tails are associated with the quantum Bose enhancement effect of identical gluons, just like the Bose-Einstein distribution of identical noninteracting bosons. Thus, we believe that, although the details of the distribution are model dependent (MV model in our case), the main feature of large fluctuations is universal as long as the fluctuations are dominated by BE.

Finally, we have also calculated the correction due to the generating function due to pairwise HBT correlations. In the regime studied in the present paper, this correction is small. However, it is bound to become important in the regime of the saturated projectile and therefore in itself would be an interesting object of study.

\section{ACKNOWLEDGMENTS}

We thank Anton Andronic, Adam Bzdak, Michael Lublinsky, Mark Mace, Larry McLerran, Prithwish Tribedy, and Urs Wiedemann for useful discussions on problems related to this project. We are especially thankful to Adrian Dumitru, Yuri Kovchegov, and R. Venugopalan for illuminating discussions. V.S. thanks Peter BraunMunzinger for questions and discussions during the EMMI Nuclear Matter and Particle seminar. This paper is an attempt to answer one of Peter's questions. This research was supported by the NSF Nuclear Theory Grant No. 1614640 and CERN scientific associateship (A. K.). V. S. is indebted to Urs Wiedemann and the CERN TH group for the partial support and hospitality at CERN, where this work was finalized. V.S. also gratefully acknowledges partial support by the ExtreMe Matter Institute EMMI (GSI Helmholtzzentrum für Schwerionenforschung, Darmstadt, Germany).

\section{APPENDIX: NUMERICS}

For numerical calculations, it is easier to work in the semifactorizable representation (see Ref. [32])

$$
\begin{aligned}
\left.\frac{d N}{d^{2} q d y}\right|_{\rho_{\mathrm{p}}, \rho_{\mathrm{t}}} & =\frac{2}{(2 \pi)^{3}} \frac{1}{|\underline{q}|^{2}}\left(\delta_{i j} \delta_{l m}+\epsilon_{i j} \epsilon_{l m}\right) \Omega_{i j}^{a}(\underline{q})\left[\Omega_{l m}^{a}(\underline{q})\right]^{*} \\
& =\frac{2}{2(2 \pi)^{3}} \frac{1}{|\underline{q}|^{2}}\left(\Omega_{\|}^{a}(\underline{q})\left[\Omega_{\|}^{a}(\underline{q})\right]^{*}+\Omega_{\perp}^{a}(\underline{q})\left[\Omega_{\perp}^{a}(\underline{q})\right]^{*}\right),
\end{aligned}
$$

where

$$
\Omega_{i j}^{a}(\underline{q})=\int d^{2} x e^{-i \underline{q} \cdot \underline{x}} \Omega_{i j}^{a}(\underline{x})
$$

and

$$
\Omega_{i j}^{a}(\underline{x})=g\left[\frac{\partial_{i}}{\partial^{2}} \rho_{\mathrm{p}}^{b}(\underline{x})\right] \partial_{j} U^{a b}(\underline{x}),
$$

with the adjoint Wilson line defined as

$$
U^{a b}(\underline{x})=2 \operatorname{tr}\left[t^{b} V^{\dagger}(\underline{x}) t^{a} V(\underline{x})\right]
$$

and

$$
\begin{aligned}
& \Omega_{\| \mid}^{a}(\underline{k})=\delta_{l m} \Omega_{l m}^{a}(\underline{k}) \equiv \Omega_{11}^{a}(\underline{k})+\Omega_{22}^{a}(\underline{k}), \\
& \Omega_{\perp}^{a}(\underline{k})=\epsilon_{l m} \Omega_{l m}^{a}(\underline{k}) \equiv \Omega_{12}^{a}(\underline{k})-\Omega_{21}^{a}(\underline{k}) .
\end{aligned}
$$

The calculations are performed on a two-dimensional lattice. For the projectile, the color sources are generated from a Gaussian ensemble, see Eq. (10), with $\mu_{\mathrm{p}} / \mu_{\mathrm{t}}=1 / 4$ and the radius of the projectile, $R_{\mathrm{p}}=1 / \mu_{\mathrm{p}}$. The Poisson equation entering in Eq. (A3), $\frac{1}{\partial^{2}} \rho_{\mathrm{p}}^{a}(\underline{x})$, is regulated by $\Lambda$, according to

$$
\frac{1}{\partial^{2}} \rho_{\mathrm{p}}^{a}(\underline{x}) \rightarrow \frac{1}{\partial^{2}-\Lambda^{2}} \rho_{\mathrm{p}}^{a}(\underline{x})
$$

For the target, we use the MV model with

$\left\langle\rho_{\mathrm{t}}^{a}\left(x^{-}, \underline{x}\right) \rho_{\mathrm{t}}^{b}\left(y^{-}, \underline{y}\right)\right\rangle_{\mathrm{t}}=\mu_{\mathrm{t}}^{2} \delta(\underline{x}-\underline{y}) \delta\left(x^{-}-y^{-}\right) \delta^{a b}$

and compute the fundamental Wilson lines

$$
V(\underline{x})=\mathbb{P} \exp \left(i g^{2} \int d x^{-} t^{a} \frac{1}{\partial^{2}} \rho_{\mathrm{t}}^{a}\left(x^{-}, \underline{x}\right)\right)
$$

Further details can be found in Refs. [33,34]. 
[1] V. Khachatryan et al. (CMS Collaboration), J. High Energy Phys. 01 (2011) 079.

[2] G. Aad et al. (ATLAS Collaboration), New J. Phys. 13, 053033 (2011).

[3] R. Aaij et al. (LHCb Collaboration), Eur. Phys. J. C 74, 2888 (2014).

[4] S. Acharya et al. (ALICE Collaboration), Eur. Phys. J. C 77, 852 (2017).

[5] B. Abelev et al. (ALICE Collaboration), Phys. Lett. B 712, 165 (2012).

[6] J. Adam et al. (ALICE Collaboration), J. High Energy Phys. 08 (2016) 078.

[7] S. G. Weber (ALICE Collaboration), Nucl. Phys. A967, 333 (2017).

[8] Y.-Q. Ma, P. Tribedy, R. Venugopalan, and K. Watanabe, arXiv:1803.11093.

[9] A. Kovner and A. H. Rezaeian, Phys. Rev. D 95, 114028 (2017).

[10] T. Altinoluk, N. Armesto, G. Beuf, A. Kovner, and M. Lublinsky, Phys. Lett. B 751, 448 (2015).

[11] T. Altinoluk, N. Armesto, G. Beuf, A. Kovner, and M. Lublinsky, Phys. Rev. D 95, 034025 (2017).

[12] A. Kovner and A. H. Rezaeian, Phys. Rev. D 96, 074018 (2017).

[13] F. Gelis, T. Lappi, and L. McLerran, Nucl. Phys. A828, 149 (2009).

[14] A. Dumitru and Y. Nara, Phys. Rev. C 85, 034907 (2012).

[15] B. Schenke, P. Tribedy, and R. Venugopalan, Phys. Rev. C 86, 034908 (2012).
[16] B. Schenke, P. Tribedy, and R. Venugopalan, Phys. Rev. C 89, 024901 (2014).

[17] A. Dumitru and E. Petreska, arXiv:1209.4105.

[18] Y. V. Kovchegov and D. E. Wertepny, Nucl. Phys. A925, 254 (2014).

[19] A. Dumitru and V. Skokov, Phys. Rev. D 96, 056029 (2017).

[20] Y. V. Kovchegov and A. H. Mueller, Nucl. Phys. B529, 451 (1998).

[21] A. Dumitru and L. D. McLerran, Nucl. Phys. A700, 492 (2002).

[22] J. P. Blaizot, F. Gelis, and R. Venugopalan, Nucl. Phys. A743, 13 (2004).

[23] A. Kovner and M. Lublinsky, J. High Energy Phys. 11 (2006) 083.

[24] T. Altinoluk, N. Armesto, and D. E. Wertepny, J. High Energy Phys. 05 (2018) 207.

[25] Y. V. Kovchegov and V. V. Skokov, Phys. Rev. D 97, 094021 (2018).

[26] L. O'Raifeartaigh, A. Wipf, and H. Yoneyama, Nucl. Phys. B271, 653 (1986).

[27] C. P. Korthals Altes, Nucl. Phys. B420, 637 (1994).

[28] A. Dumitru, G. Kapilevich, and V. Skokov, Nucl. Phys. A974, 106 (2018).

[29] E. Iancu and L. McLerran, Nucl. Phys. A793, 96 (2007).

[30] L. McLerran and P. Tribedy, Nucl. Phys. A945, 216 (2016).

[31] A. Bzdak and K. Dusling, Phys. Rev. C 93, 031901 (2016).

[32] L. McLerran and V. Skokov, Nucl. Phys. A959, 83 (2017).

[33] T. Lappi, Eur. Phys. J. C 55, 285 (2008).

[34] A. Dumitru and V. Skokov, Phys. Rev. D 91, 074006 (2015). 